\title{
A PENSION FUND IN THE ACCUMULATION PHASE: A STOCHASTIC CONTROL APPROACH
}

\author{
SALVATORE FEDERICO \\ Scuola Normale Superiore, Pisa, Italy \\ E-mail: s.federico@sns.it
}

\begin{abstract}
In this paper we propose and study a continuous time stochastic model of optimal allocation for a defined contribution pension fund in the accumulation phase. The level of wealth is constrained to stay above a "solvency level". The fund manager can invest in a riskless asset and in a risky asset, but borrowing and short selling are prohibited. The model is naturally formulated as an optimal stochastic control problem with state constraints and is treated by the dynamic programming approach. We show that the value function of the problem is a continuous viscosity solution of the associated Hamilton-Jacobi-Bellman equation. In the special case when the boundary is absorbing we show that it is the unique viscosity solution of the Hamilton-Jacobi-Bellman equation.
\end{abstract}

1. Introduction. In the paper [6] the authors propose and study a continuous time stochastic model of optimal allocation for a defined contribution pension fund with minimum guarantee.

Their target is to maximize the expected utility from the current wealth over an infinite horizon, whereas usually the portfolio selection models for pension funds maximize the expected utility from the final wealth over a finite horizon (the retirement time). In this model the dynamics of the wealth takes directly into account the flows of contributions and benefits; moreover the level of wealth is constrained to stay above a "solvency level". The fund manager can invest in a riskless asset and in a risky asset, but borrowing and short selling are prohibited. The model is formulated as an optimal stochastic control problem with constraints and is treated by the dynamic programming approach, showing that the value function of the problem is a regular solution of the associated Hamilton-Jacobi-Bellman equation. Then they apply verification techniques to get the

2000 Mathematics Subject Classification: 91B28, 93E20, 49L25.

J.E.L. classification: C61, G11, G23.

Key words and phrases: defined contribution pension fund, stochastic optimal control, dynamic programming, Hamilton-Jacobi-Bellman equations, viscosity solutions.

The paper is in final form and no version of it will be published elsewhere. 
optimal allocation strategy in feedback form and to study its properties, giving finally a special example with explicit solution. Nevertheless the aim of the authors is to study the problem starting from the time when the first retirements of contributors occur (in this case the contribution flow becomes constant and the state equation homogeneous with respect to time), leaving out the accumulation phase and the optimization problem in the first period.

In our paper we describe the model and the problem in the accumulation phase, when the state equation is time-dependent. We will show that the value function is continuous and that it solves the associated Hamilton-Jacobi-Bellman equation in a viscosity sense. When the boundary is absorbing the value function is explicitally computable on this boundary and so a Dirichlet type condition is available for the boundary differential problem. In this case we will show that it is the unique viscosity solution of the HamiltonJacobi-Bellman equation.

2. The model. In this section we give a brief survey of the model described in [6], focusing on the topics in which we are involved.

Over an infinite continuous-time model it is considered a financial market competitive $^{1}$, frictionless ${ }^{2}$, viable $^{3}$, default free ${ }^{4}$ and continuously open ${ }^{5}$.

The investor is a price taker ${ }^{6}$ and faces the following trading constraints: borrowing and short positions ${ }^{7}$ are not allowed and the pension fund wealth must be above a suitable positive function called solvency level. The investor maximizes the expected utility from the fund wealth over a finite horizon.

2.1. Demography of the fund. It is supposed a demographic stationarity hypothesis, i.e. that the flow of people who enter in the fund starts at time $t=0$ and is constant over time and that there is an exogenous constant $T>0$ which is the time during which the members adhere to the pension fund. Therefore the exit flow of people is null in the interval $[0, T]$ and is constant after time $T$, balancing exactly the entrance flow. Observe

\footnotetext{
${ }^{1}$ Investor's behavior is optimizing: he optimizes his utility function on the whole time horizon and believes his actions cannot affect the probability distribution of returns of the available assets (i.e. the investor is price taker).

${ }^{2}$ All assets are perfectly divisible and there are no transaction costs or taxes.

${ }^{3}$ There is no opportunity to gain without assuming risk with not null probability, i.e. the market is arbitrage free.

${ }^{4}$ Financial institutions issuing assets cannot default.

${ }^{5}$ The investor can continuously trade in the market: he can buy or sell assets at any time.

${ }^{6}$ The hypothesis that the investor is a price taker is usual in the literature regarding financial management models of pension funds and it is realistic if the single agent does not invest a big amount of money. As a matter of fact, the volume of assets exchanged by pension funds is such that they could affect the price of assets (i.e. the investor may be price maker), but we do not deal with this fact here.

${ }^{7}$ Borrowing position means that the investor borrows the riskless asset (paying the risk free rate of return as remuneration) to buy risky assets. In a short selling position the investor sells risky assets without owning it in his portfolio.
} 
that $T$ is not necessarily the time of retirement ${ }^{8}$. Of course there is a fixed number $N$ of fund members at time $T$ : therefore we can say that in each unit of time (e.g. year) $N / T$ new members enter in the fund.

2.2. The wealth dynamics. We focus our analysis on the accumulation phase $[0, T]$, when, by demographic hypothesis, nobody leaves the fund and so the benefits are still not paid. To set up the mathematical model consider a complete probability space $(\Omega, \mathcal{F}, P)$ on which a standard one-dimensional Brownian motion $(B(t))_{t \in[0, T]}$ is defined. The filtration $\left(\mathcal{F}_{t}\right)_{t \in[0, T]}$, representing the information available while time goes on, will be the completion of the natural filtration induced by the Brownian motion; this is a continuous filtration with respect to which $B$ is still a Brownian motion (see [12] for a detailed discussion).

The financial market is composed of two kinds of assets: a riskless asset and a risky asset.

Hypothesis 2.1. The price of the riskless asset, denoted by $S^{0}(t), t \in[0, T]$, evolves according to the equation

$$
\frac{d S^{0}(t)}{S^{0}(t)}=r d t, \quad S^{0}(0)=1,
$$

where $r \geq 0$ is the instantaneous spot rate of return.

Hypothesis 2.2. The price of the risky asset $S^{1}(t), t \in[0, T]$, follows an Itô process and satisfies the equation

$$
\frac{d S^{1}(t)}{S^{1}(t)}=\mu d t+\sigma d B(t),
$$

where $\mu \geq r$ is the instantaneous rate of expected return and $\sigma>0$ is the instantaneous rate of volatility.

The drift $\mu$ can be expressed by the relation $\mu=r+\sigma \lambda$, where $\lambda \geq 0$ is the instantaneous risk premium of the market, i.e. the price that the market assigns to the randomness expressed by the standard Brownian motion $B .{ }^{9}$ The aim is to find an optimal portfolio allocation strategy of a defined contribution pension fund.

It is also supposed that the pension fund is related to a homogeneous class of workers. ${ }^{10}$

The optimal allocation policy is determined applying the expected utility criterium: the fund manager invests the pension fund wealth between the two alternative investments maximizing his utility. Then the decision variable is represented by the proportion of wealth that the manager can invest respectively in the two assets offered by the market.

Let $X(t)$ be, for $t \in[0, T]$, the $\left(\mathcal{F}_{t}\right)_{t \in[0, T]}$-progressively measurable process that describes the amount of the pension fund wealth at any time. It is supposed the pension

\footnotetext{
${ }^{8}$ We can think that $T$ is the average time that the members spend in the fund, taking into acconut also the fraction of participants that decide to transfer their positions to another pension fund before their retirement.

${ }^{9}$ The assumption $\lambda>0$ means that the investor is risk adverse.

${ }^{10} \mathrm{~A}$ class of people that have the same characteristics (same age at the entry date, same professional qualification, same level of skill, and so on).
} 
fund starts its activity at the date $t=0$ and that at this time it owns a starting amount of wealth $x_{0}>0$. Let $\theta(t)$ be, for $t \in[0, T]$, the $\left(\mathcal{F}_{t}\right)_{t \in[0, T]^{-}}$progressively measurable process representing the proportion of fund wealth to invest in the risky asset (so that $\theta(t) \in[0,1]$ for every $t \in[0, T]$, due to the borrowing and short selling constraints). Then the dynamics of wealth is expressed by the following state equation

$$
\left\{\begin{array}{l}
d X(t)=\frac{\theta(t) X(t)}{S^{1}(t)} d S^{1}(t)+\frac{[1-\theta(t)] X(t)}{S^{0}(t)} d S^{0}(t)+c(t) d t, \\
X(0)=x_{0}
\end{array}\right.
$$

where $\theta(t) X(t) / S^{1}(t)$ and $[1-\theta(t)] X(t) / S^{0}(t)$ are respectively the quantities in the portfolio of risky and riskless asset, while the non-negative progressively measurable process $c(t)$ indicates the contribution flow at time $t \in[0, T]$.

As said in the introduction, a solvency constraint must be respected:

Hypothesis 2.3. The process $X$ describing the fund wealth is subject to the following constraint

$$
X(t) \geq l(t) \quad P \text {-a.s., } \quad \forall t \in[0, T],
$$

where the positive deterministic process $l(t), t \in[0, T]$, is a given datum which represents the solvency level.

This hypothesis is important since it prevents improper behavior of the fund manager. If this assumption is not held, he could keep the fund wealth at a too low level for long periods.

The state equation (1) can be rewritten in the following way:

$$
\left\{\begin{array}{l}
d X(t)=[(r+\sigma \lambda \theta(t)) X(t)+c(t)] d t+\sigma \theta(t) X(t) d B(t), \quad t \in[0, T], \\
X(0)=x_{0} \geq l_{0} \geq 0
\end{array}\right.
$$

with the constraint that $X(t) \geq l(t) P$-a.s., for any $t \in[0, T]$, and where $l_{0}:=l(0)$.

2.3. Contributions. The demographic hypothesis leads to assume the following:

Hypothesis 2.4. The payment of aggregate contributions occurs at any time $t \in[0, T]$ according to the following relation

$$
c(t):=\alpha N w(t) \cdot(t / T),
$$

where $\alpha \in(0,1)$ represents the average contribution rate and $w(t) \geq 0$, the average per capita wage bill earned by the fund members at time $t \in[0, T]$. The function $w(\cdot)$ is taken equal to a constant $w>0$ for simplicity and we set $k:=\frac{\alpha N w}{T}$.

The above hypothesis is a bit restrictive because the stochastic wage is an important and additional source of uncertainty for the fund manager. We observe that the introduction of an extra source of risk renders the market incomplete, as discussed and solved in [2] in absence of guarantee. Here the constant $w$ is considered as a real wage, i.e. the nominal wage discounted from a constant inflation rate, therefore we assume a point of view essentially in line with [1] where the (nominal) wage is a deterministic function of the time and continuously increasing at a constant inflation rate. 
2.4. The solvency level. Usually a solvency level is imposed by law; without imposing this constraint the fund manager is allowed to use too risky strategies for the fund.

In our case we assume that:

- at the beginning the company should hold a given minimum startup level $l_{0} \geq 0$;

- for $t \in[0, T]$, the solvency level is the capitalization at a rate $\beta \leq r^{11}$ of $l_{0}$ and of the contributions paid up to time $t$; therefore,

$$
l(t)=l_{0} e^{\beta t}+\int_{0}^{t} k s e^{\beta(t-s)} d s .
$$

REMARK 2.5. The solvency level introduced in $[6]$ is

$$
l(t)=l_{0}+\zeta \int_{0}^{t} k e^{\delta(t-s)} d u, \quad t \in[0, T],
$$

and $l(t)=l(T)$ for $t \geq T$; here $0 \leq \zeta \leq 1$ and $\delta \leq r$ is the rate corresponded to the fund members who are leaving the fund (after time $T$ ) as minimum guarantee; in this case $l_{0}$ represents only a minimum startup level for the fund to begin the financial operations, which does not need to be capitalized whereas $k$ represents the contribution rate of new members per unit of time, so that the capitalization is computed only on this term (in that context this makes the problem autonomous after time $T$ and a suitable assumption on the startup level guarantees that the fund is always able to pay the minimum guarantee to its members in retirement). We point out that almost all we prove in this paper holds also for this form of solvency level. Our solvency level seems to be more reasonable and more convenient in our setting, which focuses on the optimization problem in the interval $[0, T]$.

2.5. The objective functional. We study a finite horizon optimization problem in the interval $[0, T]$ related to an objective functional with this form:

$$
E\left[\int_{0}^{T} e^{-\rho t} U(t, X(t)) d t+f(X(T))\right] .
$$

Thus in our problem the fund manager does not care about the future of the fund after time $T$. So we concentrate our analysis on the interval $[0, T]$, when both the solvency level and the state equation are time-dependent.

3. The stochastic control problem. Now we formulate and study our problem as a stochastic optimal control problem. First of all we observe that the initial time $t=0$ has been chosen as the first time of operation of the fund. However it also makes sense to look to a pension fund that is already running after a given amount of time $s \in[0, T]$, in order to establish an optimal decision policy from $s$ on.

On the probability space of the previous section let $\left(\mathcal{F}_{t}^{s}\right)_{t \in[s, T]}$ be the completion of the filtration generated by the process $\left(B^{s}(t)\right)_{t \in[s, T]}:=(B(t)-B(s))_{t \in[s, t]}$; the control process $(\theta(t))_{t \in[s, T]}$ is an $\left(\mathcal{F}_{t}^{s}\right)$-progressively measurable process with values in $[0,1]$.

Now set an initial time $s \in[0, T]$ and a given amount of wealth $x$ at time $s$. In the interval $[0, T]$ the state equation becomes, according to (3) and with the hypotheses just

\footnotetext{
${ }^{11}$ This rate could be chosen, for example, by an authority with regard to the market's parameters.
} 
stated on the contribution term,

$$
\left\{\begin{array}{l}
d X(t)=[\theta(t) \sigma \lambda+r] X(t) d t+k t d t+\theta(t) \sigma X(t) d B(t), \quad t \in[s, T], \\
X(s)=x
\end{array}\right.
$$

this equation, for any $\left\{\mathcal{F}_{t}^{s}\right\}_{t \in[s, T]}$-progressively measurable process $\theta(\cdot)$, has a unique strong mean-square continuous solution ${ }^{12}$ on the filtered probability space $(\Omega, \mathcal{F}$, $\left\{\mathcal{F}_{t}^{s}\right\}_{t \in[s, T]}, P$ ) in the interval [0,T] (see, e.g., Problem 6.15 in [12], pp. 360-361). We denote its value at time $t \in[s, T]$ by $X(t ; s, x, \theta(\cdot))$.

For $x \geq l(s)$, the problem is to maximize, over the set of the admissible strategies, which in our framework is given by

$$
\begin{array}{r}
\Theta_{a d}(s, x)=\left\{\theta:[s, T] \times \Omega \rightarrow[0,1] \text { prog. meas. w.r.t. }\left\{\mathcal{F}_{t}^{s}\right\}_{t \in[s, T]} \mid\right. \\
X(t ; s, x, \theta(\cdot)) \geq l(t), t \in[s, T]\},
\end{array}
$$

the functional

$$
J(s, x ; \theta(\cdot))=E\left[\int_{s}^{T} e^{-\rho t} U(t, X(t ; s, x, \theta(\cdot))) d t+f(X(T ; s, x, \theta(\cdot)))\right],
$$

where $\rho>0$ is the individual discount factor and where the fund manager's utility function $U$ and the exit function $f$ satisfy the following assumptions:

Hypothesis 3.1. The utility function $U$ is such that:

(i) $U: \mathcal{C}=\left\{(s, x) \in \mathbb{R}^{2} \mid x \geq l(s)\right\} \rightarrow \mathbb{R}$.

(ii) $U(s, x)=u(x-l(s))$, where $u:[0,+\infty) \rightarrow \mathbb{R}$.

(iii) $u \in C([0,+\infty) ; \mathbb{R})$ and it is increasing and concave.

Hypothesis 3.2. The exit function $f$ is such that:

(i) $f:[l(T),+\infty) \rightarrow \mathbb{R}$.

(ii) $f \in C([l(T),+\infty) ; \mathbb{R})$ and it is increasing and concave.

REMARK 3.3. Let us give some comment on the above Hypotheses 3.1 and 3.2.

- The utility function and the exit function are defined where the wealth process $X(\cdot)$ must live.

- All utility functions of the form $u(x)=\left(x-x_{0}\right)^{\gamma} / \gamma$, for $x_{0} \leq 0, \gamma \in(0,1)$, always satisfy Hypothesis 3.1.

3.1. The set of admissible strategies. As we said, in our framework the set of admissible strategies is given by (6). We show that the set $\Theta_{a d}(s, x), s \in[0, T], x \geq l(s)$, is not empty:

Proposition 3.4. Let $s \in[0, T], x \geq l(s)$ and let $X(t):=X(t ; s, x, 0)$; then

$$
X(t)-l(t) \geq(x-l(s)) e^{r(t-s)}, \quad t \in[s, T] .
$$

In particular, for each $s \in[0, T], x \geq l(s)$, the null strategy belongs to $\Theta_{a d}(s, x)$, so that $\Theta_{a d}(s, x)$ is not empty.

\footnotetext{
${ }^{12}$ Actually the solution belongs to $C\left([s, T] ; L^{p}(\Omega, P)\right)$ for any $p \in[1,+\infty)$.
} 
Proof. Let $s \in[0, T], x \geq l(s)$ and let $X(t):=X(t ; s, x, 0)$ be the state trajectory associated with the null startegy; the dynamics of $X(\cdot)$ is given by

$$
\left\{\begin{array}{l}
d X(t)=r X(t) d t+k t d t, \\
X(s)=x
\end{array}\right.
$$

the "dynamics" of the solvency level $l(\cdot)$ is given by

$$
\left\{\begin{array}{l}
d l(t)=\beta l(t) d t+k t d t, \\
l(s)=l(s) .
\end{array}\right.
$$

The claim follows taking in account that $\beta \leq r$.

REMARK 3.5. Notice that we have to distingush the cases $\beta<r, \beta=r$. In the first case, looking at the proof of the previous proposition, we see that the null strategy, for the initial starting point $(s, l(s)), s \in[0, T)$, leads to the interior part of the set $\mathcal{C}$, so that the boundary $\{(s, l(s)), s \in[0, T]\}$ is not absorbing for the problem. In the second case this boundary is absorbing; indeed let $s \in[0, T), \theta(\cdot) \in \Theta_{a d}(s, l(s))$ and consider $X(t):=X(t ; s, l(s), \theta(\cdot))$; under the probability $\tilde{P}$ given by the Girsanov transformation the dynamics of $X(t)$ is given by

$$
\left\{\begin{array}{l}
d X(t)=r X(t) d t+k t d t+\sigma \theta(t) X(t) d \tilde{B}(t), \\
X(s)=l(s),
\end{array}\right.
$$

where $\tilde{B}(t):=B(t)+\lambda t$ is a Brownian motion under $\tilde{P}$ in the interval $[s, T]$. Since $X \in C\left([s, T] ; L^{p}(\Omega, P)\right)$ for any $p \geq 1$, we also have

$$
\tilde{E}\left[\int_{s}^{T}|X(t)|^{2} d t\right]<+\infty
$$

so that

$$
\tilde{E}\left[\int_{s}^{t} X(r) d r\right]=0, \text { for any } t \in[s, T] .
$$

Thus we can pass (8) to the expectations getting

$$
\left\{\begin{array}{l}
d \tilde{E}[X(t)]=r \tilde{E}[X(t)] d t+k t d t \\
\tilde{E}[X(s)]=l(s)
\end{array}\right.
$$

since by assumption $X(t) \geq l(t)$ for $t \in[s, T]$ and by $(9) \tilde{E}[X(t ; s, x, \theta(\cdot))]=l(t)$ for $t \in[s, T]$, we get that $X(t)=l(t)$ and that $\theta(\cdot) \equiv 0$, i.e. that the only admissible strategy starting from $(s, l(s))$ is the null one and that the corresponding state trajectory remains on the boundary, i.e. the boundary is absorbing.

3.2. The value function. The stochastic control problem consists in studying, for $s \in$ $[0, T], x \geq l(s)$, the value function

$$
V(s, x):=\sup _{\theta(\cdot) \in \Theta_{a d}(s, x)} E\left[\int_{s}^{T} e^{-\rho t} U(t, X(t ; s, x, \theta(\cdot))) d t+f(X(T ; s, x, \theta(\cdot))],\right.
$$

and, when possible, in finding an optimal pair $\left(X^{*}, \theta^{*}\right)$ (if it exists) for the problem, i.e. a strategy $\theta^{*}(\cdot) \in \Theta_{a d}(s, x)$ such that for the corresponding trajectory $X^{*}(t):=$ 
$X\left(t ; s, x, \theta^{*}(\cdot)\right)$ we have

$$
V(s, x)=J\left(s, x ; \theta^{*}(\cdot)\right)=E\left[\int_{s}^{T} e^{-\rho t} U\left(t, X^{*}(t)\right) d t+f\left(X^{*}(T)\right)\right] .
$$

It makes sense, for technical reasons, to give also a definition of $\varepsilon$-optimal strategy:

Definition 3.6. Let $s \in[0, T], x \geq l(s)$; for fixed $\varepsilon>0$, a control $\theta^{\varepsilon}(\cdot) \in \Theta_{a d}(s, x)$ is called $\varepsilon$-optimal if

$$
J\left(s, x ; \theta^{\varepsilon}(\cdot)\right) \geq V(s, x)-\varepsilon .
$$

Proposition 3.7. Let us suppose that Hypotheses 3.1 and 3.2 hold true and let $s \in[0, T]$, $x \geq l(s), \theta(\cdot) \in \Theta_{a d}(s, x)$; then setting $X(t):=X(t ; s, x, \theta(\cdot))$, we have

$$
E\left[\int_{s}^{T} e^{-\rho t}[U(t, X(t))] d t+f(X(T))\right]<+\infty .
$$

Proof. By Hypotheses 3.1-(iii), 3.2, we have, for some $C>0, U(t, x) \leq C(1+x)$ for each $t \in[s, T]$ and $f(x) \leq C(1+x)$. Thus

$$
E\left[\int_{s}^{T} e^{-\rho t}[U(t, X(t))] d t+f(X(T))\right] \leq C E\left[\int_{s}^{T} e^{-\rho t}(1+X(t)) d t+(1+X(T))\right] .
$$

Taking into account that $X(t) \geq l_{0} \geq 0$ and that $X \in C\left([s, T] ; L^{2}(\Omega)\right)$, we have

$$
E\left[\int_{s}^{t} \theta(r) X(r) d B(r)\right]=0, \quad \forall t \in[s, T] .
$$

Therefore we can pass to the expectations in the state equation getting

$$
\left\{\begin{array}{l}
d E[X(t)]=r E[X(t)] d t+k t d t+\sigma \lambda E[\theta(t) X(t)] d t \leq(r+\sigma \lambda) E[X(t)] d t+k T d t, \\
E[X(s)]=x
\end{array}\right.
$$

thus, for some $C>0$,

$$
E[X(t)] \leq\left(x+\frac{k T}{r+\sigma \lambda}\right) e^{(r+\sigma \lambda)(t-s)}-\frac{k T}{r+\sigma \lambda} \leq C(1+x) ;
$$

therefore the claim easily follows.

From the proof of Proposition 3.7 (the estimate (12) does not depend on the control) we have directly the following result:

COROLlary 3.8. There exists a constant $C>0$ such that $V(s, x) \leq C(1+x)$ for all $s \in[0, T], x \geq l(s)$.

3.3. Properties of the value function. In this section we investigate some qualitative properties of the value function. We start by analyzing the finiteness of the value function from below:

Proposition 3.9. Let $s \in[0, T], x \geq l(s)$; then

$$
V(s, x) \geq \frac{u(x-l(s))}{\rho} e^{-\rho s}\left(1-e^{-\rho(T-s)}\right)+f(l(T)+x-l(s)) .
$$


Proof. By (7) and by monotonicity of $u, f$, we can deduce that

$$
\begin{aligned}
J(s, x ; 0) & \geq \int_{s}^{T} e^{-\rho t} u(x-l(s)) d t+f(l(T)+x-l(s)) \\
& =\frac{u(x-l(s))}{\rho} e^{-\rho s}\left(1-e^{-\rho(T-s)}\right)+f(l(T)+x-l(s))
\end{aligned}
$$

and thus the claim follows.

We have also:

Proposition 3.10. Let $s \in[0, T]$; the function $x \mapsto V(s, x)$ is concave on $[l(s),+\infty)$.

Proof. Fix $x, x^{\prime} \geq l(s)$; set also $x_{\gamma}:=\gamma x+(1-\gamma) x^{\prime}, \gamma \in[0,1]$; of course $x_{\gamma} \geq l(s)$. We have to prove that

$$
V\left(s, x_{\gamma}\right) \geq \gamma V(s, x)+(1-\gamma) V\left(s, x^{\prime}\right) .
$$

Take $\theta(\cdot) \in \Theta_{a d}(s, x)$ and $\theta^{\prime}(\cdot) \in \Theta_{a d}\left(s, x^{\prime}\right) \varepsilon$-optimal for $x, x^{\prime}$ respectively and $X(\cdot), X^{\prime}(\cdot)$ the corresponding trajectories; then

$$
\begin{aligned}
\gamma V(s, x)+(1-\gamma) V\left(s, x^{\prime}\right) \leq & \gamma[J(s, x ; \theta(\cdot))+\varepsilon]+(1-\gamma)\left[J\left(s, x^{\prime} ; \theta^{\prime}(\cdot)\right)+\varepsilon\right] \\
= & \varepsilon+\gamma J(s, x ; \theta(\cdot))+(1-\gamma) J\left(s, x^{\prime} ; \theta^{\prime}(\cdot)\right) \\
= & \varepsilon+\gamma E\left[\int_{s}^{T} e^{-\rho t} U(t, X(t)) d t+f(X(T))\right] \\
& +(1-\gamma) E\left[\int_{s}^{T} e^{-\rho t} U\left(t, X^{\prime}(t)\right) d t+f\left(X^{\prime}(T)\right)\right] \\
= & \varepsilon+E\left[\int_{s}^{T} e^{-\rho t}\left[\gamma U(t, X(t))+(1-\gamma) U\left(t, X^{\prime}(t)\right)\right] d t\right] \\
& +E\left[\gamma f(X(T))+(1-\gamma) f\left(X^{\prime}(T)\right)\right] .
\end{aligned}
$$

The concavity of $u, f$ implies that

$$
\begin{gathered}
\gamma U(t, X(t))+(1-\gamma) U\left(t, X^{\prime}(t)\right) \leq U\left(t, \gamma X(t)+(1-\gamma) X^{\prime}(t)\right), \quad \forall t \in[s, T], \\
\gamma f(X(t))+(1-\gamma) f\left(X^{\prime}(t)\right) \leq f\left(\gamma X(t)+(1-\gamma) X^{\prime}(t)\right), \quad \forall t \in[s, T] .
\end{gathered}
$$

Consequently, if we set $X_{\gamma}(\cdot):=\gamma X(\cdot)+(1-\gamma) X^{\prime}(\cdot)$, we get

$$
\gamma V(s, x)+(1-\gamma) V\left(s, x^{\prime}\right) \leq \varepsilon+E\left[\int_{s}^{T} e^{-\rho t} U\left(X_{\gamma}(t)\right) d t+f\left(X_{\gamma}(T)\right)\right] .
$$

If there exists $\theta_{\gamma}(\cdot) \in \Theta\left(s, x_{\gamma}\right)$ such that $X_{\gamma}(\cdot) \leq X\left(\cdot ; s, x_{\gamma}, \theta_{\gamma}(\cdot)\right)$, then we would have

$$
\varepsilon+E\left[\int_{s}^{T} e^{-\rho t} U\left(X_{\gamma}(t)\right) d t+f\left(X_{\gamma}(T)\right)\right] \leq \varepsilon+J\left(s, x_{\gamma} ; \theta_{\gamma}(\cdot)\right) \leq \varepsilon+V\left(s, x_{\gamma}\right),
$$

i.e.

$$
\gamma V(s, x)+(1-\gamma) V\left(s, x^{\prime}\right) \leq \varepsilon+V\left(s, x_{\gamma}\right)
$$

and therefore, by the arbitrariness of $\varepsilon$, the claim (13) would be proved. We will show 
that $\theta_{\gamma}(t):=a(t) \theta(t)+d(t) \theta^{\prime}(t)$, where $a(\cdot)=\gamma \frac{X(\cdot)}{X_{\gamma}(\cdot)}$ and $d(\cdot)=(1-\gamma) \frac{X^{\prime}(\cdot)}{X_{\gamma}(\cdot)}$, is good. The admissibility of $\theta_{\gamma}$ is clear since:

(i) for any $t \in[s, T]$ we have $\theta(t), \theta^{\prime}(t) \in[0,1]$, and $a(t)+d(t)=1$, so that by convexity of $[0,1]$ we get $\theta_{\gamma}(t) \in[0,1]$;

(ii) by construction $X_{\gamma}(t) \geq l(t)$ for any $t \in[s, T]$.

Note that actually we will prove that $X_{\gamma}(\cdot)=X\left(\cdot ; s, x_{\gamma}, \theta_{\gamma}(\cdot)\right)$.

The equation satisfied by $X_{\gamma}(\cdot)$ in the interval $[s, T]$ is

$$
\begin{aligned}
d X_{\gamma}(t)= & \gamma d X(t)+(1-\gamma) d X^{\prime}(t) \\
= & \gamma[[(r+\sigma \lambda \theta(t)) X(t)+k t] d t+\sigma \theta(t) X(t) d B(t)] \\
& +(1-\gamma)\left[\left[\left(r+\sigma \lambda \theta^{\prime}(t)\right) X^{\prime}(t)+k t\right] d t+\sigma \theta^{\prime}(t) X^{\prime}(t) d B(t)\right] \\
= & {\left[r X_{\gamma}(t)+\sigma \lambda\left(\gamma \theta(t) X(t)+(1-\gamma) \theta^{\prime}(t) X^{\prime}(t)\right)+k t\right] d t } \\
& +\sigma\left[\gamma \theta(t) X(t)+(1-\gamma) \theta^{\prime}(t) X^{\prime}(t)\right] d B(t) \\
= & {\left[r X_{\gamma}(t)+k t\right] d t+\sigma \lambda\left[\gamma \theta(t) \frac{X(t)}{X_{\gamma}(t)}+(1-\gamma) \theta^{\prime}(t) \frac{X^{\prime}(t)}{X_{\gamma}(t)}\right] X_{\gamma}(t) d t } \\
& +\sigma\left[\gamma \theta(t) \frac{X(t)}{X_{\gamma}(t)}+(1-\gamma) \theta^{\prime}(t) \frac{X^{\prime}(t)}{X_{\gamma}(t)}\right] X_{\gamma}(t) d B(t) \\
= & {\left[\left(r+\sigma \lambda \theta_{\gamma}(t)\right) X_{\gamma}(t)+k t\right] d t+\sigma \theta_{\gamma}(t) X_{\gamma}(t) d B(t) }
\end{aligned}
$$

and the claim follows.

Now we can give a monotonicity result:

Proposition 3.11. Let $u$ or $f$ be strictly increasing and let $s \in[0, T]$. Then the function $x \mapsto V(s, x)$ is strictly increasing on $[l(s),+\infty)$.

Proof. Let $l(s) \leq x \leq x^{\prime}$; by comparison criterion, see e.g. Proposition 2.18 in [12], we have $X(t ; s, x, \theta(\cdot)) \leq X\left(t ; s, x^{\prime}, \theta(\cdot)\right)$ for all $\theta(\cdot) \in \Theta_{a d}(s, x)$ and in particular $\Theta_{a d}(s, x) \subset$ $\Theta_{a d}\left(s, x^{\prime}\right)$. Therefore by monotonicity of $u, f$ we get $J(s, x ; \theta(\cdot)) \leq J\left(s, x^{\prime}, \theta(\cdot)\right)$ for all $\theta(\cdot) \in \Theta_{a d}(s, x)$, so that $V(s, \cdot)$ is increasing.

Now we prove that this function is strictly increasing. We can note that, if a concave and increasing function is not strictly increasing, then such a function must be eventually constant on a right half line $[\bar{x},+\infty)$; we show that this is not our case.

If $\lim _{x \rightarrow \infty} u(x)=+\infty$ or $\lim _{x \rightarrow \infty} f(x)=+\infty$, then, by Proposition 3.9, we have also $\lim _{x \rightarrow+\infty} V(s, x)=+\infty$ and the claim follows. Instead let us suppose that $\lim _{x \rightarrow+\infty} u(x)$ $=\bar{u}<+\infty$ and $\lim _{x \rightarrow \infty} f(x)=\bar{f}<+\infty$ and suppose by contradiction that $V(s, \cdot)$ is constant on $[\bar{x},+\infty)$ for some $\bar{x} \geq l(s)$. Again by Proposition 3.9 we must have

$$
V(s, \bar{x})=\lim _{x \rightarrow+\infty} V(s, x) \geq \frac{\bar{u}}{\rho} e^{-\rho s}\left(1-e^{-\rho(T-s)}\right)+\bar{f} ;
$$

on the other hand, taking into account (12), the concavity and the monotonicity of $u, f$, 
we can write, for any $\theta(\cdot) \in \Theta_{a d}(s, \bar{x})$, setting $X(t):=X(t ; s, \bar{x}, \theta(\cdot))$,

$$
\begin{aligned}
J(s, \bar{x} ; \theta(\cdot)) & =\int_{s}^{T} e^{-\rho t} E[u(X(t)-l(t))] d t+E[f(X(T))] \\
& \leq \int_{s}^{T} e^{-\rho t} u(E[X(t)]-l(t)) d t+f(E[X(T)]) \\
& \leq \int_{s}^{T} e^{-\rho t} u(C(1+\bar{x})) d t+f(C(1+\bar{x})) \\
& =\frac{u(C(1+\bar{x}))}{\rho} e^{-\rho s}\left(1-e^{-\rho(T-s)}\right)+f(C(1+\bar{x})),
\end{aligned}
$$

i.e. since $u$ or $f$ is strictly increasing,

$$
\begin{aligned}
V(s, x) & \leq \frac{u(C(1+\bar{x}))}{\rho} e^{-\rho s}\left(1-e^{-\rho(T-s)}\right)+f(C(1+\bar{x})) \\
& <\frac{\bar{u}}{\rho} e^{-\rho s}\left(1-e^{-\rho(T-s)}\right)+\bar{f}
\end{aligned}
$$

thus a contradiction arises and the claim is proved.

3.4. Continuity of the value function. In this section we will prove that the value function is continuous on $\mathcal{C}=\left\{(s, x) \in \mathbb{R}^{2} \mid x \geq l(s)\right\}$; we will prove this result by some lemmata. LEMmA 3.12. Let $s \in[0, T], \varepsilon>0$; the function $[l(s)+\varepsilon,+\infty) \rightarrow[0,+\infty), x \mapsto V(s, x)$ is Lipschitz continuous.

Proof. The claim follows by the fact that the function $[l(s),+\infty) \rightarrow \mathbb{R}, x \mapsto V(s, x)$ is concave and increasing. This implies that this function is continuous in the interior part of its domain $(l(s),+\infty)$ and Lipschitz continuous on $[l(s)+\varepsilon,+\infty)$ for any $\varepsilon>0$.

Let us define, for $a \geq 0$, the curves

$$
\mathcal{L}_{a}:=\{(s, l(s)+a) \mid s \in[0, T]\} .
$$

LEMMA 3.13. Let $u(0) \geq 0, a \geq 0$; then the value function is decreasing along the curve $\mathcal{L}_{a}$, i.e. the function $[0, T] \rightarrow \mathbb{R}, s \mapsto V(s, l(s)+a)$ is decreasing.

Proof. Let $s \in[0, T], s^{\prime} \in(s, T]$ and let $x, x^{\prime}$ be such that $(s, x),\left(s^{\prime}, x^{\prime}\right) \in \mathcal{L}_{a}$, for some $a \geq 0$; let us consider $X(t):=X(t ; s, x, 0)$; we get

$$
V(s, x) \geq E\left[\int_{s}^{s^{\prime}} e^{-\rho t} U(t, X(t)) d t\right]+V\left(s^{\prime}, X\left(s^{\prime}\right)\right) \geq V\left(s^{\prime}, x^{\prime}\right)
$$

where the first inequality follows by Dynamic Programming Principle (see Theorem 4.1 and Remark 4.2), the second one follows taking into account (7), which gives that $X\left(s^{\prime}\right) \geq$ $x^{\prime}$ and that the utility function $u$ is positive, and by Proposition 3.11.

REMARK 3.14. Let $0 \leq s \leq s^{\prime} \leq T$; by Theorem 2.10 (chapter 1 ) of [18] we can map in a natural way a strategy starting at time $s$ to a strategy starting at time $s^{\prime}$; to make this point more clear, consider the measurable space $(C[s, T], \mathcal{B}(C[s, T]))$, with the filtration $\left(\mathcal{B}_{t}(C[s, T])\right)_{t \in[s, T]}$ defined in the following way: $\left(\mathcal{B}_{t}(C[s, T])\right)$ is the $\sigma$-algebra on $C[s, T]$ induced by the projection

$$
\pi: C[s, T] \rightarrow(C[s, t], \mathcal{B}(C[s, t])),\left.\quad \zeta(\cdot) \mapsto \zeta(\cdot)\right|_{[s, t]},
$$


i.e. the smallest $\sigma$-algebra which makes $\pi$ measurable; intuitively a measurable map with respect to $\mathcal{B}_{t}(C[s, T])$ is a map which does not distinguish between two functions of $C[s, T]$ which coincide on $[s, t]$. If $\left(\theta_{s}(t)\right)_{t \in[s, T]}$ is a strategy starting from $s$, there exists a process $\psi$ on $(C[s, T], \mathcal{B}(C[s, T]))$, adapted with respect to $(\mathcal{B}(C[s, T]))_{t \in[s, T]}$ such that

$$
\theta_{s}(t)=\psi\left(t, B^{s}(\cdot)\right), \quad t \in[s, T]
$$

then we can consider the strategy

$$
\theta_{s^{\prime}}(t)=\psi\left(t-s^{\prime}+s, B^{s^{\prime}}(\cdot)\right), \quad t \in\left[s^{\prime}, T\right],
$$

starting from $s^{\prime}$; we denote by $\Gamma_{s, s^{\prime}}$ the map $\theta_{s} \mapsto \theta_{s^{\prime}}$.

The following lemma is the crucial key to proving the continuity of the value function:

LEMmA 3.15. Let $u(0) \geq 0$; then the value function is continuous along the curves $\mathcal{L}_{a}$ for any $a \geq 0$.

Proof. Fix $a \geq 0$ and $s \in[0, T)$, let $s^{\prime} \in(s, T]$ and let $x, x^{\prime}$ be such that $(s, x),\left(s^{\prime}, x^{\prime}\right) \in$ $\mathcal{L}_{a}$, i.e $x^{\prime}-x=l\left(s^{\prime}\right)-l(s)$; take a generic control $\theta_{s}(\cdot) \in \Theta_{a d}(s, x)$, set $\varepsilon:=s^{\prime}-s$ and consider, for $t \in[s, T]$, the process $X_{s}(t):=X\left(t ; s, x, \theta_{s}(\cdot)\right)$ and, for $t \in\left[s^{\prime}, T\right]$, the process $Y_{s^{\prime}}(t)$ given by

$$
\left\{\begin{array}{l}
d Y_{s^{\prime}}(t)=\left(r+\sigma \lambda \theta_{s^{\prime}}(t)\right) Y_{s^{\prime}}(t) d t+k(t-\varepsilon) d t+\sigma \theta_{s^{\prime}}(t) Y_{s^{\prime}}(t) d B(t), \\
Y_{s^{\prime}}\left(s^{\prime}\right)=x,
\end{array}\right.
$$

where $\theta_{s^{\prime}}(\cdot)=\Gamma_{s, s^{\prime}}\left(\theta_{s}(\cdot)\right)$. Of course $X_{s}(t-\varepsilon) \stackrel{\mathcal{L}}{=} Y_{s^{\prime}}(t)$ and, by the assumption $\theta(\cdot) \in$ $\Theta_{a d}(s, x)$, we get $Y_{s^{\prime}}(t) \geq l(t-\varepsilon)$ on $\left[s^{\prime}, T\right]$ almost surely. Define the "semi-feedback" strategy $\tilde{\theta}_{s^{\prime}}(\cdot)$ starting from $s^{\prime}$ by

$$
\tilde{\theta}_{s^{\prime}}(t)=\theta_{s^{\prime}}(t) \frac{Y_{s^{\prime}}(t)}{X_{s^{\prime}}(t)},
$$

where $X_{s^{\prime}}(\cdot)$ denotes the solution of the state equation starting from $x^{\prime}$ at time $s^{\prime}$ under the strategy $\tilde{\theta}_{s^{\prime}}(\cdot)$; we will show that $\tilde{\theta}_{s^{\prime}}(\cdot)$ takes values in $[0,1]$ and that $\tilde{\theta}_{s^{\prime}}(\cdot) \in$ $\Theta_{a d}\left(s^{\prime}, x^{\prime}\right)$. The dynamics of $X_{s^{\prime}}(\cdot)-Y_{s^{\prime}}(\cdot)$ is given by

$$
\left\{\begin{array}{l}
d\left(X_{s^{\prime}}(t)-Y_{s^{\prime}}(t)\right)=r\left(X_{s^{\prime}}(t)-Y_{s^{\prime}}(t)\right) d t+\varepsilon k d t \\
X_{s^{\prime}}\left(s^{\prime}\right)-Y_{s^{\prime}}\left(s^{\prime}\right)=l\left(s^{\prime}\right)-l(s) ;
\end{array}\right.
$$

the dynamics for $l(t)-l(t-\varepsilon)$ is given by

$$
\left\{\begin{array}{l}
d(l(t)-l(t-\varepsilon))=\beta(l(t)-l(t-\varepsilon)) d t+\varepsilon k d t \\
l\left(s^{\prime}\right)-l\left(s^{\prime}-\varepsilon\right)=l\left(s^{\prime}\right)-l(s) ;
\end{array}\right.
$$

comparing (14), (15) and taking into account that $\beta \leq r$, we get

$$
X_{s^{\prime}}(t)-Y_{s^{\prime}}(t) \geq l(t)-l(t-\varepsilon) ;
$$

this shows that $\tilde{\theta}_{s^{\prime}}(\cdot)$ takes values in the set $[0,1]$ and, since $Y_{s^{\prime}}(t) \geq l(t-\varepsilon)$, that $\theta_{s^{\prime}}(\cdot) \in \Theta\left(s^{\prime}, x^{\prime}\right)$.

We have proved that $X_{s^{\prime}}(t) \geq Y_{s^{\prime}}(t) \stackrel{\mathcal{L}}{=} X_{s}(t-\varepsilon)$, for $t \in\left[s^{\prime}, t\right]$; thus, taking into account that $u$ is increasing and uniformly continuous, if $\delta$ is the modulus of uniform 
continuity of $u$ and $\delta^{\prime}$ is the modulus of uniform continuity of $l$, we have

$$
\begin{aligned}
E\left[\int_{s^{\prime}}^{T} e^{-\rho t} U\left(t, X_{s^{\prime}}(t)\right) d t\right] & \geq E\left[\int_{s^{\prime}}^{T} e^{-\rho t} U\left(t, X_{s}(t-\varepsilon)\right) d t\right] \\
& =E\left[\int_{s}^{T-\varepsilon} e^{-\rho(t+\varepsilon)} U\left(t+\varepsilon, X_{s}(t)\right) d t\right] \\
& =e^{-\rho \varepsilon} E\left[\int_{s}^{T-\varepsilon} e^{-\rho t} u\left(X_{s}(t)-l(t+\varepsilon)\right) d t\right] \\
& \geq e^{-\rho \varepsilon} E\left[\int_{s}^{T-\varepsilon} e^{-\rho t} u\left(X_{s}(t)-l(t)-\delta^{\prime}(\varepsilon)\right) d t\right] \\
& \geq e^{-\rho \varepsilon} E\left[\int_{s}^{T-\varepsilon} e^{-\rho t} u\left(X_{s}(t)-l(t)\right) d t\right]-C \delta\left(\delta^{\prime}(\varepsilon)\right),
\end{aligned}
$$

for a suitable constant $C>0$, where $\delta\left(\delta^{\prime}(\varepsilon)\right) \rightarrow 0$, when $\varepsilon \rightarrow 0$.

Of course, by the square-mean continuity of $X_{s}$ and by the uniform continuity of $u$,

$$
E\left[\int_{T-\varepsilon}^{T} e^{-\rho t} U\left(t, X_{s}(t)\right) d t\right] \rightarrow 0
$$

In the same way

$$
E\left[f\left(X_{s^{\prime}}(T)\right)\right] \geq E\left[f\left(X_{s}(T-\varepsilon)\right)\right]
$$

moreover, by the mean-square continuity of $X_{s}$ and by the uniform continuity of $f$,

$$
E\left[\left|f\left(X_{s}(T-\varepsilon)\right)-f\left(X_{s}(T)\right)\right|^{2}\right] \rightarrow 0,
$$

when $\varepsilon \rightarrow 0$, so that, combining (18) and (19),

$$
E\left[f\left(X_{s^{\prime}}(T)\right)\right] \geq E\left[f\left(X_{s}(T)\right)\right]-\eta(\varepsilon),
$$

with $\eta(\varepsilon) \rightarrow 0$, when $\varepsilon \rightarrow 0$.

Fix $s \in[0, T]$; combining (16), (17) and (20), for any $\varepsilon>0$ and any control $\theta_{s}(\cdot) \in$ $\Theta_{a d}(s, x)$, we can find a control $\tilde{\theta}_{s^{\prime}}(\cdot) \in \Theta_{a d}\left(s^{\prime}, x^{\prime}\right)$ such that

$$
J\left(s^{\prime}, x^{\prime} ; \tilde{\theta}_{s^{\prime}}(\cdot)\right) \geq e^{-\rho \varepsilon} J\left(s, x ; \theta_{s}(\cdot)\right)-\omega(\varepsilon),
$$

where $\omega(\varepsilon) \rightarrow 0$, when $\varepsilon \rightarrow 0$; this is enough to guarantee that the value function is lower semicontinuous from the right along $\mathcal{L}_{a}$ and, since it is decreasing by Lemma 3.13, that it is continuous from the right. Instead, if we fix $s^{\prime}$, by the above argument, for any $\varepsilon>0$ and any control $\theta_{s}(\cdot) \in \Theta_{a d}(s, x)$, we can find a control $\tilde{\theta}_{s^{\prime}}(\cdot) \in \Theta_{a d}\left(s^{\prime}, x^{\prime}\right)$ such that $(21)$ holds with $\omega(\varepsilon) \rightarrow 0$, when $\varepsilon \rightarrow 0$; this shows that the value function is upper semicontinuous from the left along $\mathcal{L}_{a}$ and, again since it is decreasing, continuous from the left and so the proof is complete.

Let us define, for $\varepsilon>0$, the sets

$$
S_{\varepsilon}:=\left\{(s, x) \in \mathbb{R}^{2} \mid x \geq l(s)+\varepsilon\right\} .
$$

Lemma 3.16. Let $u(0) \geq 0$; then the value function is continuous on the sets $S_{\varepsilon}$ for any $\varepsilon>0$.

Proof. Let $s \in[0, T], \varepsilon>0$ and consider the function $[l(s)+\varepsilon) \rightarrow \mathbb{R}, x \mapsto V(s, x)$; by Lemma 3.12 this function is Lipschitz continuous: we want to estimate its Lipschitz 
constant. The function $[l(s),+\infty) \rightarrow \mathbb{R}, x \mapsto V(s, x)$ is concave (so that the incremental ratios are decreasing) and increasing (so that the incremental ratios are positive); thus, if we set

$$
M_{s, \varepsilon}:=\frac{V(s, l(s)+\varepsilon)-V(s, l(s))}{\varepsilon},
$$

we get that $M_{s, \varepsilon}$ is good as Lipschitz constant for the function $[l(s)+\varepsilon,+\infty) \rightarrow \mathbb{R}$, $x \mapsto V(s, x)$. By Lemma 3.15 there exists

$$
M_{\varepsilon}:=\max _{s \in[0, T]} M_{s, \varepsilon}=\max _{s \in[0, T]} \frac{V(s, l(s)+\varepsilon)-V(s, l(s))}{\varepsilon} ;
$$

thus the functions $[l(s)+\varepsilon,+\infty) \rightarrow[0,+\infty), x \mapsto V(s, x), s \in[0, T]$, are Lipschitz continuous with respect the same Lipschitz constant $M_{\varepsilon}$. Thus, for any $\delta>0$, we can find $\eta>0$ such that, if $|x-y|<\eta$ (with $x, y \in[l(s)+\varepsilon,+\infty)$ ), then $|V(s, x)-V(s, y)|<\delta / 2$ and $\eta$ does not depend on $s \in[0, T]$, due to the uniform Lipschitz continuity. Now fix $(\bar{s}, \bar{x}) \in S_{2 \varepsilon}$ and define $\bar{x}(s):=l(s)+\bar{x}-l(\bar{s})$, so that $s \mapsto \bar{x}(s)$ is the curve $\mathcal{L}_{a}$ passing through $(\bar{s}, \bar{x})$ and through $\bar{x}(\bar{s})=\bar{x}$. By the continuity of $s \mapsto V(s, \bar{x}(s))$, for a given $\delta>0$, we can find $\eta^{\prime}>0$ such that, if $|s-\bar{s}|<\eta^{\prime}$, then $|V(s, \bar{x}(s))-V(\bar{s}, \bar{x})|<\delta / 2$. Therefore, for a given $\delta>0$, we can find $\eta, \eta^{\prime}>0$ such that, if

$$
(s, x) \in \bigcup_{s \in\left(\bar{s}-\eta^{\prime}, \bar{s}+\eta^{\prime}\right)}\{s\} \times(\bar{x}(s)-\eta, \bar{x}(s)+\eta),
$$

then $|V(s, x)-V(\bar{s}, \bar{x})|<\delta$ and so the claim is proved.

Lemma 3.17. Let $u(0) \geq 0, s \in[0, T]$; the function $[l(s),+\infty) \rightarrow[0+\infty), x \mapsto V(s, x)$ is continuous at $l(s)$.

Proof. Of course the function is lower semicontinuous at $l(s)$, since it is increasing; we will prove that it is also upper semicontinuous at $l(s)$. We have to distinguish the two cases when the boundary is absorbing or not, i.e. when $\beta<r$ or $\beta=r$ (see Remark 3.5).

CASE 1: $\beta<r$. Let $s \in(0, T]$ and $s^{\prime} \in[0, s)$; consider $X_{s^{\prime}}(t):=X\left(t ; s^{\prime}, l\left(s^{\prime}\right), 0\right)$; then the function $t \mapsto V\left(t, X_{s^{\prime}}(t)\right)$ is decreasing by Dynamic Programming Principle (Theorem 4.1), since $u$ is positive; moreover, looking at the proof of Proposition 3.4, we see that $X_{s^{\prime}}(s)>l(s)$, due to the assumption $\beta<r$, and that $X_{s^{\prime}}(s) \downarrow l(s)$, when $s^{\prime} \uparrow s$, because of continuous dependence on the initial datum $s^{\prime}$ of the state equation. Consider also the value function along $\mathcal{L}_{0}$ in the time interval $\left[s^{\prime}, s\right]$; by Lemma 3.15 it is continuous; thus

$$
\limsup _{x \downarrow l(s)} V(s, x)=\limsup _{s^{\prime} \uparrow s} V\left(s, X_{s^{\prime}}(s)\right) \leq \limsup _{s^{\prime} \uparrow s} V\left(s^{\prime}, l\left(s^{\prime}\right)\right)=V(s, l(s)),
$$

where the inequality holds since $t \mapsto V\left(t, X_{s^{\prime}}(t)\right)$ is decreasing and the last equality holds since the value function is continuous along $\mathcal{L}_{0}$; therefore the claim is proved for $s \in(0, T]$. In the case $s=0$, we can argue as well as before by extending also for $s<0$ the problem with $k=0$, setting the solvency level $l(s) \equiv l_{0}$ and defining the value function in the obvious way.

CASE 2: $\beta=r$. In this case we proceed directly with estimates on the state equation. Let $x>l(s), \varepsilon>0$ and let $D$ be the density of $P$ with respect to the probability measure $\tilde{P}$ given by the Girsanov transformation, which belongs to $L^{p}(\Omega, \tilde{P})$, for any $p \in[1,+\infty)$. 
For any $\theta(\cdot) \in \Theta_{a d}(s, x), t \in[s, T]$, we have, by the Hölder and Markov inequalities

$$
\begin{aligned}
P\{X(t ; s, x, \theta(\cdot))-l(t)>\varepsilon\} & =E\left[I_{\{X(t ; s, x, \theta(\cdot))-l(t)>\varepsilon\}}\right]=\tilde{E}\left[I_{\{X(t ; s, x, \theta(\cdot))-l(t)>\varepsilon\}} D\right] \\
& \leq\left(\tilde{E}\left[D^{2}\right]\right)^{1 / 2}\left(\tilde{E}\left[I_{\{X(t ; s, x, \theta(\cdot))-l(t)>\varepsilon\}}\right]\right)^{1 / 2} \\
& =\left(\tilde{E}\left[D^{2}\right]\right)^{1 / 2}(\tilde{P}\{X(t ; s, x, \theta(\cdot))-l(t)>\varepsilon\})^{1 / 2} \\
& \leq \frac{\left(\tilde{E}\left[D^{2}\right]\right)^{1 / 2}}{\varepsilon^{1 / 2}}(\tilde{E}[X(t ; s, x, \theta(\cdot))-l(t)])^{1 / 2} .
\end{aligned}
$$

Let us estimate $\tilde{E}[X(t ; s, x, \theta(\cdot))-l(t)]$; we have, arguing as in the proof of Proposition 3.4,

$$
\left\{\begin{array}{l}
d \tilde{E}[X(t)]=r X(t) d t+k t d t, \\
\tilde{E}[X(s)]=x,
\end{array}\right.
$$

and

$$
\left\{\begin{array}{l}
d l(t)=r l(t) d t+k t d t \\
l(s)=l(s)
\end{array}\right.
$$

so that

$$
\tilde{E}[X(t ; s, x, \theta(\cdot))-l(t)]=(x-l(s)) e^{r(t-s)} ;
$$

let $\left(\varepsilon_{n}, \delta_{n}\right)$ be a sequence; we have shown that, for any $n \in \mathbb{N}$, we can find $x_{n}$ such that $l(s)<x_{n}<l(s)+1 / n$ and

$$
P\left\{X\left(t ; s, x_{n}, \theta(\cdot)\right)-l(t)>\varepsilon_{n}\right\}<\delta_{n}, \quad \forall \theta(\cdot) \in \Theta_{a d}\left(s, x_{n}\right), \forall t \in[s, T] .
$$

Moreover we can estimate $E\left[(X(t ; s, x, \theta(\cdot))-l(t))^{2}\right]$ uniformly with respect to $t, x, \theta(\cdot)$ varying in the sets $[s, T],[l(s), l(s)+1], \Theta_{a d}(s, x)$; indeed, by the Dynkin formula applied with the function $\psi(t, x)=(x-l(t))^{2}$, we have

$$
\begin{aligned}
E\left[(X(t ; s, x, \theta(\cdot))-l(t))^{2}\right]= & (x-l(s))^{2}+E\left[-2 \int_{s}^{t}(X(r)-l(r)) l^{\prime}(r) d r\right] \\
& +E\left[2 \int_{s}^{t}(X(r)-l(r))((r+\sigma \lambda \theta(r)) X(r)+k r) d r\right] \\
& +E\left[\int_{s}^{t} \sigma^{2} \theta(r)^{2} X(r)^{2} d r\right]
\end{aligned}
$$

and the right-hand side is dominated by a constant $C$ (not dependent on $t \in[s, T]$, $\left.x \in[l(s), l(s)+1], \theta(\cdot) \in \Theta_{a d}(s, x)\right)$ by the mean-square continuity of $X(\cdot)$ and since $\theta(\cdot) \in[0,1]$. Thus we can split the expectation and write, again by the Hölder inequality,

$$
\begin{aligned}
E\left[X\left(t ; s, x_{n}, \theta(\cdot)\right)-l(t)\right] & \leq \varepsilon_{n}+E\left[I_{\left\{X\left(t ; s, x_{n}, \theta(\cdot)\right)-l(t)>\varepsilon_{n}\right\}}\left(X\left(t ; s, x_{n}, \theta(\cdot)\right)-l(t)\right)\right] \\
& \leq \varepsilon_{n}+C \delta_{n}^{1 / 2},
\end{aligned}
$$

for some constant $C$ not dependent on $n$; therefore, for such a point $x_{n}>l(s)$, it follows, by the concavity and the monotonicity of $u, f$ and by the Jensen inequality, that

$$
V\left(s, x_{n}\right) \leq \int_{s}^{T} u\left(\varepsilon_{n}+C \delta_{n}^{1 / 2}\right) d t+f\left(\varepsilon_{n}+C \delta_{n}^{1 / 2}+l(T)\right) .
$$

If we take $\left(\varepsilon_{n}, \delta_{n}\right)$ such that $\left(\varepsilon_{n}, \delta_{n}\right) \rightarrow 0$ when $n \rightarrow \infty$, the right-hand side in the previous inequality tends to $V(s, l(s))$ and so the claim is proved. 
LEMma 3.18. Let $u(0) \geq 0$; then the value function is continuous on $\mathcal{C}$.

Proof. It remains only to prove the continuity at the boundary (on the interior part it was proved in Lemma 3.16). By Lemma 3.17 we know that

$$
V(s, l(s)+\varepsilon) \downarrow V(s, l(s)), \quad \text { for any } s \in[0, T],
$$

(when $\varepsilon \downarrow 0$ ) and moreover, by Lemma 3.15, we know that $s \mapsto V(s, l(s)$ ) is continuous. Therefore by Dini's lemma, $V(\cdot, l(\cdot)+\varepsilon) \rightarrow V(\cdot, l(\cdot))$ uniformly when $\varepsilon \downarrow 0$. This convergence, together with the continuity of $s \mapsto V(s, l(s))$, is enough to prove the claim.

In order to conclude we have to remove the assumption $u(0) \geq 0$ :

Proposition 3.19. The value function is continuous on $\mathcal{C}$.

Proof. Let $u(0)=-c<0$; consider the function $u^{c}(\cdot):=u(\cdot)+c$ and let $V^{c}$ be the value function associated with this utility function; by Lemma $3.18 V^{c}$ is continuous on $\mathcal{C}$. Moreover $V(s, x)=V^{c}(s, x)-\frac{c}{\rho}\left(e^{-\rho s}-e^{-\rho T}\right)$ and so the claim follows.

4. Dynamic programming. We study the optimization problem following a dynamic programming approach. The core of the dynamic programming is the Dynamic Programming Principle, which can be stated as follows:

THEOREM 4.1. The value function $V$ satisfies the dynamic programming equation, i.e. for every $s \in[0, T], x \in[l(s),+\infty)$ and $\tau \in[s, T]$ a stopping time, the following functional equation holds true:

$$
V(s, x)=\sup _{\theta(\cdot) \in \Theta_{a d}(s, x)} \mathbb{E}\left[\int_{s}^{\tau} e^{-\rho t} U(t, X(t ; s, x, \theta(\cdot))) d t+V(\tau, X(\tau ; s, x, \theta(\cdot)))\right] .
$$

REMARK 4.2. We do not give the proof of the previous theorem, but we want to comment about it: in [18], Chapter 4, Theorem 3.3, there is a proof of this statement when the value function is continuous: therein the state is unconstrained, but the argument can be easily adapted to our case. A general proof of this statement, where the continuity of the value function is not required, is contained in [17]; it requires a measurable selection result.

However we want to point out that we have proved the continuity of our value function using in Lemma 3.13 and in Lemma 3.17 only the inequality

$V(s, x) \geq \int_{s}^{s^{\prime}} e^{-\rho t} U(t, X(t ; s, x, 0)) d t+V\left(s^{\prime}, X\left(s^{\prime} ; s, x, 0\right)\right), \quad 0 \leq s \leq s^{\prime} \leq T, x \geq l(s)$,

which can be proved without any measurable selection argument, because in this case we are on a deterministic trajectory. Therefore we can use the argument of [18] in order to prove the Dynamic Programming Principle without loss of generality.

In general the dynamic programming equation is hard to treat. Then one usually studies its differential form, i.e. the Hamilton-Jacobi-Bellman (hereafter HJB) equation. We introduce the following Hamiltonian function

$$
H(s, x, p, Q)=\sup _{\theta \in[0,1]} H_{c v}(s, x, p, Q ; \theta), \quad s \in[0, T], \quad x \in[l(s),+\infty), \quad p, Q \in \mathbb{R},
$$


where

$$
H_{c v}(s, x, p, Q ; \theta)=e^{-\rho s} U(s, x)+p([\theta \sigma \lambda+r] x+k s)+\frac{1}{2} \theta^{2} \sigma^{2} x^{2} Q
$$

The HJB equation on the domain $\mathcal{C}$ associated with our problem is then the following one:

$$
\begin{cases}-v_{s}(s, x)-H\left(s, x, v_{x}(s, x), v_{x x}(s, x)\right)=0, & (s, x) \in \stackrel{\circ}{\mathcal{C}} \\ v(T, x)=f(x), & x \in[l(T),+\infty)\end{cases}
$$

calling

$$
H_{c v}^{0}(x, p, Q ; \theta)=\theta \sigma \lambda x p+\frac{1}{2} \theta^{2} \sigma^{2} x^{2} Q
$$

we can write

$$
H(s, x, p, Q)=e^{-\rho s} U(s, x)+p(r x+k s)+\sup _{\theta \in[0,1]} H_{c v}^{0}(x, p, Q ; \theta) .
$$

To calculate the Hamiltonians we can observe that the function

$$
H_{c v}^{0}(x, p, Q ; \theta)=p \theta \sigma \lambda x+\frac{1}{2} \theta^{2} \sigma^{2} x^{2} Q,
$$

when $p \geq 0, Q \leq 0, p^{2}+Q^{2}>0$, has a unique maximum point over $\theta \in[0,1]$ given by

$$
\theta^{*}=-\frac{\lambda p}{\sigma x Q} \wedge 1
$$

(where we mean that, for $Q=0, \theta^{*}=1$ ) and

$$
H^{0}(x, p, Q)=\sup _{\theta \in[0,1]} H_{c v}^{0}(x, p, Q ; \theta)= \begin{cases}-\frac{\lambda^{2} p^{2}}{2 Q}, & \text { if } \quad \theta^{*}<1, \\ p \sigma \lambda x+\frac{1}{2} \sigma^{2} x^{2} Q, & \text { if } \quad \theta^{*}=1 .\end{cases}
$$

When $p=Q=0$ each $\theta \in[0,1]$ is a maximum point and $H^{0}(x, p, Q)=0$.

4.1. The HJB equation: viscosity solutions. Let us consider HJB equation $(24)$ on $\mathcal{C}$. Let us define the sets

$$
\operatorname{Int}^{*}(\mathcal{C}):=\operatorname{Int}(\mathcal{C}) \cup\left\{\{0\} \times\left(l_{0},+\infty\right)\right\}, \quad \partial^{*} \mathcal{C}:=\{(s, x) \in \mathcal{C} \mid s \in[0, T), x=l(s)\} .
$$

Next we give the definition of viscosity sub(super)-solution on $\operatorname{Int}^{*}(\mathcal{C})$ and of viscosity subsolution on $\partial^{*} \mathcal{C}$ (see, e.g., [4] for a survey on viscosity solutions of second order PDE's).

Definition 4.3. (i) A continuous function $v: \mathcal{C} \rightarrow \mathbb{R}$ is called a viscosity subsolution (respectively supersolution) of the HJB equation $(24)$ on $\operatorname{Int}^{*}(\mathcal{C})$ if $v(T, x) \leq f(x)$ (respectively $v(T, x) \geq f(x))$, for $x \in[l(T),+\infty)$, and if, for any couple $\psi \in C^{2}(\mathcal{C} ; \mathbb{R})$ and $\left(s_{M}, x_{M}\right) \in \operatorname{Int}^{*}(\mathcal{C})$ such that $\left(s_{M}, x_{M}\right)$ is a local maximum for $v-\psi$ on $\operatorname{Int}^{*}(\mathcal{C})$ (respectively $\left(s_{m}, x_{m}\right) \in \operatorname{Int}^{*}(\mathcal{C})$ such that $\left(s_{m}, x_{m}\right)$ is a local minimum for $v-\psi$ on $\left.\operatorname{Int}^{*}(\mathcal{C})\right)$, we have

$$
-\psi_{s}\left(s_{M}, x_{M}\right)-H\left(s_{M}, x_{M}, \psi_{x}\left(s_{M}, x_{M}\right), \psi_{x x}\left(s_{M}, x_{M}\right)\right) \leq 0 .
$$

(respectively

$$
\left.-\psi_{s}\left(s_{m}, x_{m}\right)-H\left(s_{m}, x_{m}, \psi_{x}\left(s_{m}, x_{m}\right), \psi_{x x}\left(s_{m}, x_{m}\right)\right) \geq 0 .\right)
$$

(ii) A continuous function $v: \mathcal{C} \rightarrow \mathbb{R}$ is called a viscosity solution of the HJB equation (24) on $\operatorname{Int}^{*}(\mathcal{C})$ if it is both a viscosity subsolution and a viscosity supersolution on $\operatorname{Int}(\mathcal{C})$. 
(iii) A continuous function $v: \mathcal{C} \rightarrow \mathbb{R}$ is called a viscosity subsolution of the HJB equation $(24)$ on $\partial^{*} \mathcal{C}$ if $v(T, x) \leq f(x)$, for $x \in[l(T),+\infty)$, and if, for any couple $\psi \in C^{2}(\mathcal{C} ; \mathbb{R})$ and $\left(s_{M}, x_{M}\right) \in \partial^{*} \mathcal{C}$ such that $\left(s_{M}, x_{M}\right)$ is a local maximum for $v-\psi$ on $\mathcal{C}$, we have

$$
-\psi_{s}\left(s_{M}, x_{M}\right)-H\left(s_{M}, x_{M}, \psi_{x}\left(s_{M}, x_{M}\right), \psi_{x x}\left(s_{M}, x_{M}\right)\right) \leq 0 .
$$

(iv) A continuous function $v: \mathcal{C} \rightarrow \mathbb{R}$ is called a constrained viscosity solution of the HJB equation $(24)$ on $\mathcal{C}$ if it is a viscosity solution on $\operatorname{Int}^{*}(\mathcal{C})$ and a viscosity subsolution on $\partial^{*} \mathcal{C}$.

Now we can state and prove the following result:

THEOREM 4.4. The value function $V$ is a constrained viscosity solution of the HJB equation $(24)$ on $\mathcal{C}$.

Proof. (i) Let $\psi \in C^{2}(\mathcal{C} ; \mathbb{R})$ and let $\left(s_{m}, x_{m}\right) \in \operatorname{Int}^{*}(\mathcal{C})$ be such that $\left(s_{m}, x_{m}\right)$ is a local minimum point for $V-\psi$. We can assume without loss of generality that

$$
V\left(s_{m}, x_{m}\right)=\psi\left(s_{m}, x_{m}\right) \quad \text { and } \quad V(s, x) \geq \psi(s, x), \quad \forall(s, x) \in \mathcal{C} .
$$

Let $\theta \in[0,1]$ and set $X(t):=X\left(t ; s_{m}, x_{m}, \theta\right)$. Let us define $\tau^{\theta}=\inf \left\{t \geq s_{m} \mid(t, X(t)) \notin\right.$ Int $\left.t^{*}(\mathcal{C})\right\}$, with the convention $\inf \emptyset=T$; of course $\tau^{\theta}$ is a stopping time and $\tau^{\theta}>s_{m}$ almost surely. By $(25)$ we get, for any $t \in\left[s_{m}, \tau^{\theta}\right]$,

$$
V(t, X(t))-V\left(s_{m}, x_{m}\right) \geq \psi(t, X(t))-\psi\left(s_{m}, x_{m}\right) .
$$

Let $h \in\left(s_{m}, T\right]$ and set $\tau_{h}^{\theta}:=\tau^{\theta} \wedge h$; by the dynamic programming principle (22) we get, for any $\theta \in[0,1]$,

$$
\begin{aligned}
0 & \geq E\left[\int_{s_{m}}^{\tau_{h}^{\theta}} e^{-\rho t} U(t, X(t)) d t+V\left(\tau_{h}^{\theta}, X\left(\tau_{h}^{\theta}\right)\right)-V\left(s_{m}, x_{m}\right)\right] \\
& \geq E\left[\int_{s_{m}}^{\tau_{h}^{\theta}} e^{-\rho t} U(t, X(t)) d t+\psi\left(\tau_{h}^{\theta}, X\left(\tau_{h}^{\theta}\right)\right)-\psi\left(s_{m}, x_{m}\right)\right] .
\end{aligned}
$$

Applying the Dynkin formula to the function $\psi(t, x)$ with the process $X(t)$, we get

$$
\begin{array}{r}
E\left[\psi\left(\tau_{h}^{\theta}, X\left(\tau_{h}^{\theta}\right)\right)-\psi\left(s_{m}, x_{m}\right)\right]=E\left[\int _ { s _ { m } } ^ { \tau _ { h } ^ { \theta } } \left[\psi_{s}(t, X(t))+[(r+\sigma \lambda \theta) X(t)+k t] \psi_{x}(t, X(t)) .\right.\right. \\
\left.\left.+\frac{1}{2} \sigma^{2} \theta^{2} X(t)^{2} \psi_{x x}(t, X(t))\right] d t\right]
\end{array}
$$

and thus by (26) we have

$$
\begin{aligned}
0 \geq E\left[\int _ { s _ { m } } ^ { \tau _ { h } ^ { \theta } } \left[e^{-\rho t} U(t, X(t)) d t+\psi_{s}(t, X(t))+[(r+\right.\right. & \sigma \lambda \theta) X(t)+k t] \psi_{x}(t, X(t)) \\
& \left.\left.+\frac{1}{2} \sigma^{2} \theta^{2} X(t)^{2} \psi_{x x}(t, X(t))\right] d t\right]
\end{aligned}
$$

Thus, for any $\theta \in[0,1]$, we get

$$
0 \geq E\left[\int_{s_{m}}^{\tau_{h}^{\theta}}\left[\psi_{s}(t, X(t))+H_{c v}\left(t, X(t), \psi_{x}(t, X(t)), \psi_{x x}(t, X(t)) ; \theta\right)\right] d t\right]
$$


thus we can write, for $\theta \in[0,1]$,

$$
0 \geq E\left[\frac{1}{h-s_{m}} \int_{s_{m}}^{h} I_{\left[s_{m}, \tau^{\theta}\right]}(t)\left[\psi_{s}(t, X(t))+H_{c v}\left(t, X(t), \psi_{x}(t, X(t)), \psi_{x x}(t, X(t)) ; \theta\right)\right] d t\right] ;
$$

now, by the continuity properties of $\psi$ and $H_{c v}$, passing to the limit for $h \rightarrow s_{m}$, we get by dominated convergence

$$
-\psi_{s}\left(s_{m}, x_{m}\right)-H_{c v}\left(s_{m}, x_{m}, \psi^{\prime}\left(s_{m}, x_{m}\right), \psi^{\prime \prime}\left(s_{m}, x_{m}\right) ; \theta\right) \geq 0 .
$$

By the arbitrariness of $\theta$ we have proved that $V$ is a supersolution on $\operatorname{Int}^{*}(\mathcal{C})$.

(ii) Let $\psi \in C^{2}(\mathcal{C} ; \mathbb{R})$ and $\left(s_{M}, x_{M}\right) \in \operatorname{Int} t^{*}(\mathcal{C})$ such that $\left(s_{M}, x_{M}\right)$ is a local maximum point for $V-\psi$. We can assume, without loss of generality, that

$$
V\left(s_{M}, x_{M}\right)=\psi\left(s_{M}, x_{M}\right) \quad \text { and } \quad V(s, x) \leq \psi(s, x), \quad \forall(s, x) \in \mathcal{C} .
$$

We must prove that

$$
-\psi_{s}\left(s_{M}, x_{M}\right)-H\left(s_{M}, x_{M}, \psi_{x}\left(s_{M}, x_{M}\right), \psi_{x x}\left(s_{M}, x_{M}\right)\right) \leq 0 .
$$

Let us suppose by contradiction that this relation is false. Then there exists $\nu>0$ such that

$$
0<\nu<-\psi_{s}\left(s_{M}, x_{M}\right)-H\left(s_{M}, x_{M}, \psi_{x}\left(s_{M}, x_{M}\right), \psi_{x x}\left(s_{M}, x_{M}\right)\right)
$$

By the continuity properties of $U, \psi, H$, there exists $\varepsilon>0$ such that, if $(t, x) \in B:=$ $B\left(\left(s_{M}, x_{M}\right), \varepsilon\right) \cap \operatorname{Int}^{*}(\mathcal{C})$, we have, for any $\theta \in[0,1]$,

$$
\begin{aligned}
0<\frac{\nu}{2} & <-\psi_{s}(s, x)-H\left(s, x, \psi_{x}(s, x), \psi_{x x}(s, x)\right) \\
& \leq-\psi_{s}(s, x)-H_{c v}\left(s, x, \psi_{x}(s, x), \psi_{x x}(s, x) ; \theta\right)
\end{aligned}
$$

Let us consider any admissible control strategy $\theta(\cdot) \in \Theta_{a d}\left(s_{M}, x_{M}\right)$ and let $X(t):=$ $X\left(t ; s_{M}, x_{M}, \theta(\cdot)\right)$. Define the stopping time $\tau^{\theta}:=\inf \left\{t \geq s_{M} \mid(t, X(t)) \notin B\right\}$, with the convention $\inf \emptyset=T$; of course $\tau^{\theta}>s_{M}$ almost surely. Now we can apply (28) to $X(t)$, for $t \in\left[s_{M}, \tau^{\theta}\right]$, getting

$$
0<\frac{\nu}{2}<-\psi_{s}(t, X(t))-H_{c v}\left(t, X(t), \psi_{x}(t, X(t)), \psi_{x x}(t, X(t)) ; \theta(t)\right) ;
$$

integrating (29) on $\left[s_{M}, \tau^{\theta}\right]$ and taking the expectations we get

$0<\frac{\nu}{2} E\left[\tau^{\theta}-s_{M}\right] \leq-E\left[\int_{s_{M}}^{\tau^{\theta}} \psi_{s}(t, X(t))+H_{c v}\left(t, X(t), \psi_{x}(t, X(t)), \psi_{x x}(t, X(t)) ; \theta(t)\right) d t\right] ;$

we claim that there exists a constant $\delta>0$, independent of the control $\theta(\cdot)$, such that $\frac{\nu}{2} E\left[\tau^{\theta}-s_{M}\right] \geq \delta$; we will prove this fact in the lemma below. Thus, assuming the claim, we can write, for any $\theta(\cdot) \in \Theta_{a d}\left(s_{M}, x_{M}\right)$,

$$
\delta \leq-E\left[\int_{s_{M}}^{\tau^{\theta}}\left(\psi_{s}(t, X(t))+H_{c v}\left(t, X(t), \psi_{x}(t, X(t)), \psi_{x x}(t, X(t)) ; \theta(t)\right)\right) d t\right]
$$

applying the Dynkin formula to $X$ on $\left[s_{M}, \tau^{\theta}\right]$ we get

$$
\psi\left(s_{M}, x_{M}\right)-E\left[\psi\left(\tau^{\theta}, X\left(\tau^{\theta}\right)\right)\right] \geq \delta+E\left[\int_{s_{M}}^{\tau^{\theta}} e^{-\rho t} U(t, X(t)) d t\right]
$$


from (27) we get

$$
V\left(s_{M}, x_{M}\right)-E\left[V\left(\tau^{\theta}, X\left(\tau^{\theta}\right)\right)\right] \geq \delta+E\left[\int_{s_{M}}^{\tau^{\theta}} e^{-\rho t} U(t, X(t)) d t\right]
$$

on the other hand, if we choose a $\delta / 2$ optimal control $\theta(\cdot) \in \Theta_{a d}\left(s_{M}, x_{M}\right)$, we get

$$
V\left(s_{M}, x_{M}\right)-\delta / 2 \leq E\left[\int_{s_{M}}^{\tau^{\theta}} e^{-\rho t} U(t, X(t)) d t+V\left(\tau^{\theta}, X\left(\tau^{\theta}\right)\right)\right] .
$$

So a contradiction arises and we have proved that $V$ is a subsolution on $\operatorname{Int}(\mathcal{C})$.

(iii) Let $\psi \in C^{2}(\mathcal{C}) \rightarrow \mathbb{R}$ and $\left(s_{M}, x_{M}\right) \in \partial^{*} \mathcal{C}$ such that $\left(s_{M}, x_{M}\right)$ is a local maximum in $\mathcal{C}$ for $V-\psi$. Let us suppose by contradiction that, for some $\nu>0$,

$$
\nu<-\psi_{s}\left(s_{M}, x_{M}\right)-H\left(s_{M}, x_{M}, \psi_{x}\left(s_{M}, x_{M}\right), \psi_{x x}\left(s_{M}, x_{M}\right)\right) .
$$

By the continuity properties of $U, \psi, H$, there exists $\varepsilon>0$ such that, for any $(t, x) \in$ $B\left(\left(s_{M}, x_{M}\right), \varepsilon\right) \cap \mathcal{C}$, we have

$$
0<\frac{\nu}{2}<-\psi_{s}(t, x)-H\left(t, x, \psi_{x}(t, x), \psi_{x x}(t, x)\right) .
$$

Then, arguing as in (ii), it is possible to prove that this leads to a contradiction and therefore that $V$ is also a viscosity subsolution on $\partial^{*} \mathcal{C}$.

LEMMA 4.5. Let $\tau^{\theta}$ be the stopping time defined in the part (ii) of the proof of the previous theorem; then there exists $\delta>0$ such that, for each $\theta(\cdot) \in \Theta_{a d}\left(s_{M}, x_{M}\right)$,

$$
E\left[\tau^{\theta}-s_{M}\right] \geq \delta .
$$

Proof. For $\theta(\cdot) \in \Theta_{a d}\left(s_{M}, x_{M}\right)$, let $X(t):=X\left(t ; s_{M}, x_{M}, \theta(\cdot)\right)$ and apply the Dynkin formula to the process $X(\cdot)$ with the function $\varphi(t, x)=\left(t-s_{M}\right)^{2}+\left(x-x_{M}\right)^{2}$ on $\left[s_{M}, \tau^{\theta}\right]$; we get

$$
\begin{aligned}
& E\left[\left(\tau^{\theta}-s_{M}\right)^{2}+\left(X\left(\tau^{\theta}\right)-x_{M}\right)^{2}\right] \\
& \quad=E\left[\int_{s_{M}}^{\tau^{\theta}}\left[2\left(t-s_{M}\right)+2\left(X(t)-x_{M}\right)[(r+\sigma \lambda \theta(t)) X(t)+k t]+\sigma^{2} \theta(t)^{2} X(t)^{2}\right] d t\right] .
\end{aligned}
$$

So, considering that $\tau^{\theta} \leq T$ and $\theta(t) \in[0,1]$ and that, for $t \in\left[s_{M}, \tau^{\theta}\right]$, we have $|X(t)| \leq$ $x_{M}+\varepsilon$, we can find $K>0$ such that

$$
\left(T-s_{M}\right)^{2} \wedge \varepsilon^{2} \leq P\left\{\tau^{\theta}=T\right\}\left(T-s_{M}\right)^{2}+P\left\{\tau^{\theta}<T\right\} \varepsilon^{2} \leq E\left[\int_{s_{M}}^{\tau^{\theta}} K d t\right]=K E\left[\tau^{\theta}-s_{M}\right]
$$

this estimate does not depend on $\theta(\cdot)$ and therefore the claim is proved.

REMARK 4.6. In the definition 4.3 of constrained viscosity solution we could replace the requirement that $V-\psi$ has a local maximum (resp. minimum) at $\left(s_{M}, x_{M}\right.$ ) (resp. $\left(s_{m}, x_{m}\right)$ ) with the requirement that it has a right (with respect to the time variable) local maximum (resp. local minimum) at $\left(s_{M}, x_{M}\right)\left(\operatorname{resp} .\left(s_{m}, x_{m}\right)\right)$, i.e., for some $\varepsilon>0$,

$$
\begin{aligned}
& V\left(s_{M}, x_{M}\right)-\psi\left(s_{M}, x_{M}\right) \geq V(s, x)-\psi(s, x) \\
& \qquad \text { for }(s, x) \in\left\{\left[s_{M}, s_{M}+\varepsilon\right] \times\left[x_{M}-\varepsilon, x_{M}+\varepsilon\right]\right\} \cap \mathcal{C}
\end{aligned}
$$


(resp. the analogous for the minimum). Then we could prove exactly as in the proof of Theorem 4.4 that $V$ is a constrained viscosity solution also in this stronger sense ${ }^{13}$.

4.2. The HJB equation: comparison and uniqueness. The definition 4.3 of constrained viscosity solution which we have given is the natural version in the parabolic case of a quite standard definition of constrained viscosity solution for HJB elliptic equations arising in optimal control problems with infinite time horizon and state constraints. In particular the condition in Definition 4.3-(iii) plays the role of a boundary condition. This boundary condition was introduced by Soner in [16] in the deterministic case. In the stochastic case it was used by Katsoulakis in [13], Zariphopoulou in [20] and Ishii \& Loreti in [11]. For the study of viscosity solutions of second order fully nonlinear equations with boundary conditions see [10]. In particular in [16] and [11] this boundary condition turns out to be strong enough to guarantee, under a cone-like condition for the state equation at the boundary (see in particular assumption (A4) in [11]), the uniqueness for the solution of the HJB equation. The natural version of this cone-like condition in the parabolic context holds true in our case when $\beta<r$; for this reason we have chosen this boundary condition for the equation. However in the cited references the optimal control problem is time-homogeneous and over an infinite time-horizon, so that the associated PDE problem is elliptic. Our problem is instead strongly time-dependent, because both the state equation and the state constraint depend on time, and this leads to a parabolic PDE problem. To our knowledge a comparison principle for parabolic equations with this kind of boundary condition still misses in the literature. There are instead many results in the case of Dirichlet type condition at the boundary and we use a result contained in [9] to show in a particular case that our value function is the unique viscosity solution of $(24)$ on $\operatorname{Int}^{*}(\mathcal{C})$, satifying a Dirichlet type condition at the boundary.

Now let $\beta=r$; as we showed in Remark 3.5, in this case the boundary is absorbing and the only admissible strategy for the initial point $(s, l(s)), s \in[0, T)$, is the null one; thus in this case the value function is explicitly computable on the "lateral" boundary $\partial^{*} \mathcal{C}$, i.e. $V(s, l(s))=g(s)$, where $g:[0, T] \rightarrow \mathbb{R}$ is a known function.

Notice that, if $u, f$ are bounded, then $V$ is obviously bounded. Moreover it is straightforward to see that, thanks to Proposition 3.9, $V$ is uniformly continuous.

TheOREm 4.7. Let $\beta=r$, let $u$ be Lipschitz continuous and let $u, f$ be bounded. Then the value function $V$ is the unique bounded and uniformly continuous viscosity solution of $(24)$ on $\operatorname{Int}^{*}(\mathcal{C})$ which satisfies the boundary condition $v(s, l(s))=g(s), s \in[0, T]$.

Proof. We only sketch the proof. The complete proof can be found in [8]. We transform the equation in order to simplify the term corresponding to the drift of the state equation and make the constraint nicer (not time-dependent). So let $v$ be a bounded and uniformly continuous viscosity solution of $(24)$ on $\operatorname{Int}^{*}(\mathcal{C})$ and define $w:[0, T] \times\left[l_{0},+\infty\right) \rightarrow \mathbb{R}$ by

$$
w(t, x):=v(t, h(t, x))
$$

\footnotetext{
${ }^{13}$ The test functions $\psi$ on which the HJB equation is tested are in this case more numerous than in the case of Definition 4.3.
} 
where

$$
h(t, x):=x e^{r t}+\int_{0}^{t} k s e^{r(t-s)} d s .
$$

It is straightforward to prove, taking into account that $l^{\prime}(t)=r l(t)+k t$, that $v$ is a bounded and uniformly continuous viscosity solution of $(24)$ on $\operatorname{Int}^{*}(\mathcal{C})$ with lateral boundary condition $v(t, l(t))=g(t), t \in[0, T]$, and terminal boundary condition $v(T, x)=$ $f(x)$ if and only if $w$ is a bounded viscosity solution on $[0, T) \times\left(l_{0},+\infty\right)$, with lateral boundary condition $w\left(t, l_{0}\right)=g(t), t \in[0, T]$, and terminal boundary condition $w(T, x)=$ $f(h(T, x))$, of

$$
-u_{t}(t, x)-\tilde{H}\left(t, x, u_{x}(t, x), u_{x x}(t, x)\right)=0,
$$

where

$$
\tilde{H}(t, x, p, Q):=e^{-\rho t} U(t, h(t, x))+H^{0}(t, x, p, Q), \quad t \in[0, T), x \in\left(l_{0},+\infty\right), p, Q \in \mathbb{R} .
$$

Therefore uniqueness for bounded and uniformly continuous viscosity solutions of (24) on $\operatorname{Int}^{*}(\mathcal{C})$ holds with lateral boundary condition $v(t, l(t))=g(t), t \in[0, T]$, and terminal boundary condition $v(T, x)=f(x)$, if and only if uniqueness for bounded and uniformly continuous viscosity solutions of $(31)$ on $[0, T) \times\left(l_{0},+\infty\right)$ holds with lateral boundary condition $w\left(t, l_{0}\right)=g(t), t \in[0, T]$, and terminal boundary condition $w(T, x)=f(h(T, x))$.

Now the setting is very similar to Theorem V.8.1 in [9], but here we have to work with an unbounded domain. So the proof is a suitable modification of the proof of Theorem V.8.1 of [9]. The main feature of this modification consists in adding to the test function $\Phi$ (appearing in the cited reference) the term $-\varepsilon \log (x+y+1)$.

5. Conclusions. We have partially investigated a problem strictly related to that one studied in [6]. In the context of [6] the problem was homogeneous over time and with an infinite time horizon, therefore it was possible to get rid of the time variable and to reduce the problem to an elliptic one. Here there are some intrinsic difficulties related to the dependence on the time variable of the state equation and of the state constraint, so that the problem has higher difficulties. The main feature of this work is the proof of the continuity of the value function in this different context; this proof required a new argument with respect to that used to prove the continuity of the value function in [6]. Instead the argument used to prove that the value function is a viscosity solution of the HJB equation is a suitable modification of that one contained in [6]. The uniqueness result when the boundary is absorbing is essentially a consequence of [9]. Of course the aim of future works is to improve the study of the problem proving a uniqueness result in the case $\beta<r$ and proving regularity properties for the value function (which again would require more subtle arguments than the ones used in [6]) in order to be able to prove a verification theorem giving optimal feedback strategies also in this case.

Acknowledgements. I wish to thank in a special way my advisor, Prof. Fausto Gozzi, for careful reading of this work and for useful suggestions for setting it out in a better way. I wish to thank also the referee for the correction of some mistakes and, especially, for his suggestion on the suitable form of the solvency level. 


\section{References}

[1] J. F. Boulier, S. J. Huang and G. Taillard, Optimal management under stochastic interest rates: the case of a protected pension fund, Insurance: Mathematics and Economics 28 (2001), 173-189.

[2] A. Cairns, D. Blake and K. Dowd, Optimal dynamic asset allocation for definedcontribution pension plans, Proceedings of the 10th AFIR International Colloquium, 2000, $131-154$.

[3] I. Capuzzo Dolcetta and P. L. Lions, Viscosity solutions of Hamilton-Jacobi equations and state constraints, Transactions of the American Mathematical Society 318 (1990), 643-683.

[4] M. Crandall, H. Ishii and P. L. Lions, User's guide to viscosity solutions of second order partial differential equations, Bulletin of the American Mathematical Society (New Series) 27 (1992), 1-67.

[5] G. Deelstra, M. Grasselli and P. F. Koehl, Optimal design of a guarantee for defined contribution funds, Journal of Economic Dynamics and Control 28 (2001), 2239-2260.

[6] M. Di Giacinto and F. Gozzi, Pension funds with a minimum guarantee: a stochastic control approach, Finance and Stochasticts, submitted, 2008.

[7] S. Federico, A pension fund model with surplus: an infinite dimensional stochastic control approach, 2007, Preprint of the Scuola Normale Superiore.

[8] S. Federico, Modelli di Fondi Pensione, PhD Thesis, Scuola Normale Superiore, Pisa, 2008, work in progress.

[9] V. H. Fleming and H. M. Soner, Controlled Markov Processes and Viscosity Solutions, Springer-Verlag, New York, 1993.

[10] H. Ishii and P. L. Lions, Viscosity solutions of fully nonlinear second order partial differential equations, Journal of Differential Equations 83 (1990), 26-78.

[11] H. Ishii and P. Loreti, A class of stochastic optimal control problems with state constraint, Indiana University Mathematics Journal 5 (2002), 1167-1196.

[12] I. Karatzas and S. E. Shreve, Brownian Motion and Stochastic Calculus, 2nd ed., Springer, New York, 1991.

[13] M. Katsoulakis, Viscosity solutions of second order fully nonlinear equations with state constaints, Indiana University Mathematics Journal 43 (1994).

[14] J. M. Larsy and P. L. Lions, Nonlinear elliptic equations with singular boundary conditions and state constraints. I. The Model Problem, Math. Ann. 283 (1989), 583-630.

[15] D. Revuz and M. Yor, Continuous Martingales and Brownian motion, 3rd ed., Springer, Berlin-Heidelberg, 1999.

[16] H. M. Soner, Optimal control with state constraints, SIAM Journal of Control and Optimization 24 (1986), 552-561.

[17] H. M. Soner, Stochastic Optimal Control in Finance, Cattedra Galileiana, Scuola Normale Superiore, Pisa, 2004.

[18] J. Yong and X. Y. Zhou, Stochastic Controls: Hamiltonian Systems and HJB Equations, Springer Verlag, New York, 1999.

[19] T. Zariphopoulou, Optimal Investment-Consumption Models with Constraints, PhD Thesis, Brown University, 1985.

[20] T. Zariphopoulou, Consumption-Investment Models with Constraints, SIAM Journal on Control and Optimization 32 (1994), 59-85. 
